\title{
Forestation in hypergraphs: linear $k$-trees
}

\author{
Ojas Parekh \\ Department of Mathematics and Computer Science \\ Emory University, Atlanta, USA \\ ojas@mathcs . emory.edu
}

Submitted: Sep 14, 2001; Accepted: Sep 1, 2003; Published: Sep 23, 2003

MR Subject Classifications: 05C65, 05E99

\begin{abstract}
We present a new proof of a result of Lovász on the maximum number of edges in a $k$-forest. We also apply a construction used in our proof to generalize the notions of a $k$-hypertree and $k$-forest to a class which extends some properties of trees, to which both specialize when $k=2$.
\end{abstract}

\section{Introduction}

Let $X=[n]$ and $\mathcal{F}$ be a $k$-uniform hypergraph on $X$. We say an edge $e \in \mathcal{F}$ crosses a $k$-partition, $X=X_{1} \cup \dot{\cup} X_{k}$, if $\left|e \cap X_{i}\right|=1$ for $1 \leq i \leq k$. $\mathcal{F}$ is a $k$-forest if for each $e \in \mathcal{F}$ there is some $k$-partition $X=X_{1}^{e} \dot{\cup} \cdots \dot{\cup} X_{k}^{e}$ such that $e$ is the unique edge crossing it. What is the maximum number of edges in $\mathcal{F}$ ?

This problem was initially posed to László Lovász by Ronald Graham [2]. Lovász's novel algebraic proof appeared in [3] in 1979, and our proof remains algebraic in nature; however, it relies on homogeneous multilinear polynomials over $\mathbb{F}_{2}$ rather than tensors. The reader is encouraged to consult [1] for an introduction to and extensive applications of linear algebra in combinatorics.

Theorem 1.1. A k-forest $\mathcal{F}$ on $X$ has at most $\left(\begin{array}{c}n-1 \\ k-1\end{array}\right)$ edges.

Proof. We open with a few definitions. By $\mathbb{P}_{k-1}^{n-1}$ we mean the space of multilinear homogeneous polynomials of degree $k-1$ in $\mathbb{F}_{2}\left[x_{1}, \ldots, x_{n-1}\right]$. We make use of the shorthand $p(\mathbf{x})$ to denote $p\left(x_{1}, \ldots, x_{n-1}\right)$, where $\mathbf{x}=\left(x_{1}, \ldots, x_{n-1}\right) \in \mathbb{F}_{2}^{n-1}$ and $p \in \mathbb{P}_{k-1}^{n-1}$. Finally, for $e \in \mathcal{F}, \mathbb{1}_{e}$ denotes the incidence vector of $e$.

For each edge $e \in \mathcal{F}$ we pick a $k$-partition $\pi_{e}=\left(X_{1}^{e}, \ldots, X_{k}^{e}\right)$, such that $e$ is the unique edge crossing it. For simplicity we assume $X_{1}^{e}$ contains the element $n$. We then define a polynomial,

$$
p_{e}\left(x_{1}, \ldots, x_{n-1}\right)=\prod_{i=2}^{k} \sum_{j \in X_{i}^{e}} x_{j} .
$$


For each $e$ in $\mathcal{F}, p_{e}$ is in $\mathbb{P}_{k-1}^{n-1}$, hence it suffices to demonstrate the independence of these polynomials. To that end we seek to show that if $e, f \in \mathcal{F}$, then $p_{e}\left(\mathbb{1}_{f \backslash\{n\}}\right)=1$ if and only if $f=e$. We have

$$
p_{e}\left(\mathbb{1}_{f \backslash\{n\}}\right)=\prod_{i=2}^{k}\left(\left|f \cap X_{i}^{e}\right| \bmod 2\right) .
$$

Clearly $p_{e}\left(\mathbb{1}_{e \backslash\{n\}}\right)=1$. If $f \neq e$ there must be some $i$ for which $\left|f \cap X_{i}^{e}\right|=0$, since $f$ does not cross $\pi_{e}$. In this case there also exists a $j \neq i$ such that $\left|f \cap X_{j}^{e}\right| \bmod 2=0$. Thus $p_{e}\left(\mathbb{1}_{f \backslash\{n\}}\right)=0$.

Our agenda for the remainder of the paper is to first consider a generalization of $k$ forests which preserves certain properties of forests and to then proceed to compare our generalization with existing ones.

\section{Linear $k$-trees}

In light of the result of the previous section a natural question arises. What can one say about the maximum $k$-forests, those with exactly $\left(\begin{array}{l}n-1 \\ k-1\end{array}\right)$ edges? We could begin by considering small examples. It is not difficult to verify that a 2 -forest is indeed a forest. In this case any maximal forest is a tree, which one may define in several ways. A basic result in graph theory is that a graph which exhibits any two of

(i) acyclicity

(ii) exactly $n-1$ edges

(iii) connectivity

necessarily exhibits the third.

We already have analogues of (i) and (ii) that we could use in defining a $k$-tree for $k>2$, and one might conjecture that for a $k$-uniform hypergraph $\mathcal{H}$ on $X$, any two of

(i') $\mathcal{H}$ is a $k$-forest.

(ii') $\mathcal{H}$ has exactly $\left(\begin{array}{l}n-1 \\ k-1\end{array}\right)$ edges.

(iii') For each $k$-partition of $X, \mathcal{H}$ contains an edge that crosses it.

implies the third. Unfortunately this is not true.

Counterexample 2.1. The 3-uniform hypergraph

$$
\mathcal{H}=\{\{1,2,4\},\{1,2,5\},\{1,3,5\},\{1,4,5\},\{2,3,4\},\{3,4,5\}\}
$$

over $\{1,2,3,4,5\}$ satisfies (ii') and (iii') but not (i'). 
Why does this generalization fail? Conditions (i')-(iii') extend the notion of a cut in a graph, which is implicit in (i)-(iii), to that of a $k$-partition. We do have that a 2-partition is indeed a cut; however, this is not the whole story. The proof of Theorem 1.1 offers some insight into the matter. The multilinear polynomial space $\mathbb{P}_{1}^{n-1}$ consists entirely of polynomials which correspond to 2-partitions; however, the reader may verify that an analogous statement is not true for even $\mathbb{P}_{2}^{n-1}$. Guided by this discrepancy, we say an edge $e \in \mathcal{H}$ crosses a polynomial $p \in \mathbb{P}_{k-1}^{n-1}$ if $p\left(\mathbb{1}_{e \backslash\{n\}}\right)=1$, and we $\operatorname{relax}\left(\mathrm{i}^{\prime}\right)$ : The hypergraph $\mathcal{H}$ is a linear $k$-forest (vs. a $k$-forest) if for each edge $e \in \mathcal{H}$, there is a polynomial $p_{e} \in \mathbb{P}_{k-1}^{n-1}$ (vs. a $k$-partition) such that $e$ is the unique edge in $\mathcal{H}$ crossing $p_{e}$. We accordingly strengthen (iii'): The hypergraph $\mathcal{H}$ is linearly $k$-connected, or simply $k$-connected, if for each polynomial $p \in \mathbb{P}_{k-1}^{n-1}$, there is an edge $e \in \mathcal{H}$ which crosses $p$. The scrutinizing reader might have sensed something amiss in the preceding definitions. The polynomial space $\mathbb{P}_{k-1}^{n-1}$ is defined with respect to a distinguished element $n \in X$.

Lemma 2.2. A hypergraph is a linear $k$-forest or $k$-connected independently of the choice of distinguished element used in defining $\mathbb{P}_{k-1}^{n-1}$.

Proof. Let $p\left(x_{1}, \ldots, x_{n-1}\right) \in \mathbb{P}_{k-1}^{n-1}$. We will demonstrate a $p^{\prime}\left(x_{1}, \ldots, x_{i-1}, x_{i+1}, \ldots, x_{n}\right) \in$ $\mathbb{P}_{k-1}^{n}$ such that $\left\{e \in\left(\begin{array}{l}X \\ k\end{array}\right) \mid p\left(\mathbb{1}_{e \backslash\{n\}}\right)=1\right\}=\left\{e \in\left(\begin{array}{l}X \\ k\end{array}\right) \mid p^{\prime}\left(\mathbb{1}_{e \backslash\{i\}}\right)=1\right\}$. We divide $p$ by $x_{i}$ to yield $p=x_{i} q+r$ where $q \in \mathbb{P}_{k-2}^{n-1}, r \in \mathbb{P}_{k-1}^{n-1}$, and neither contain the variable $x_{i}$. We can represent $q$ as a sum of monomials, that is there exist sets $Y_{s} \in\left(\begin{array}{c}X \backslash\{i, n\} \\ k-2\end{array}\right)$ for $s$ in some index set $S$ such that $q=\sum_{s \in S} \prod_{j \in Y_{s}} x_{j}$. Notice that an edge crosses the polynomial $\left(\prod_{j \in Y_{s}} x_{j}\right)\left(\sum_{j \notin Y_{s} \cup\{i\}} x_{j}\right)$ if and only if it crosses the monomial $x_{i}\left(\prod_{j \in Y_{s}} x_{j}\right)$. This provides us the construction we seek, and we set

$$
p^{\prime}\left(x_{1}, \ldots, x_{i-1}, x_{i+1}, \ldots, x_{n}\right)=r+\sum_{s \in S}\left(\prod_{j \in Y_{s}} x_{j}\right)\left(\sum_{j \notin Y_{s} \cup\{i\}} x_{j}\right) .
$$

We will henceforth use $\mathbb{P}_{k-1}^{n-1}$ to refer to a multilinear polynomial space in $n-1$ variables, the indices of which will be clear from context. We now have the following.

Theorem 2.3. For $\mathcal{H}$, a $k$-uniform hypergraph on $X$, any two of

(i) $\mathcal{H}$ is a linear $k$-forest.

(ii) $\mathcal{H}$ has exactly $\left(\begin{array}{l}n-1 \\ k-1\end{array}\right)$ edges.

(iii) $\mathcal{H}$ is k-connected.

implies the third. 
Proof.

(i),(ii) implies (iii): For each edge $e$ let $p_{e}$ be a polynomial for which $e$ is the unique edge in $\mathcal{H}$ crossing $p_{e}$. For a polynomial $p \in \mathbb{P}_{k-1}^{n-1}$, let $\mathcal{H}(p)$ denote $\{e \in \mathcal{H} \mid e$ crosses $p\}$. As in the proof of Theorem 1.1 we have the independence of the polynomials $p_{e}$ for $e \in \mathcal{H}$, hence $\left|\left\{p_{e} \mid e \in \mathcal{H}\right\}\right|=\left(\begin{array}{l}n-1 \\ k-1\end{array}\right)$ by (ii). The set $\left\{p_{e} \mid e \in \mathcal{H}\right\}$ is a basis for $\mathbb{P}_{k-1}^{n-1}$, so for any $q \in \mathbb{P}_{k-1}^{n-1}$ we must have $\mathcal{H}(q) \neq \emptyset$.

(ii),(iii) implies (i): First we establish $p \neq q$ implies $\mathcal{H}(p) \neq \mathcal{H}(q)$, for polynomials $p, q \in \mathbb{P}_{k-1}^{n-1}$. Proceeding by contrapositive, if $\mathcal{H}(p)=\mathcal{H}(q)$ then $\mathcal{H}(p+q)=\emptyset$, hence $p=q$. There are exactly $2^{\left(\begin{array}{c}n-1 \\ k-1\end{array}\right)}-1$ polynomials in $\mathbb{P}_{k-1}^{n-1}$ and $|\mathcal{H}|=\left(\begin{array}{c}n-1 \\ k-1\end{array}\right)$, so by (iii) $\left\{\mathcal{H}(p) \mid p \in \mathbb{P}_{k-1}^{n-1}\right\}=2^{\mathcal{H}} \backslash\{\emptyset\}$, where $2^{\mathcal{H}}$ is the powerset of $\mathcal{H}$. Thus for each edge $e \in \mathcal{H},\{e\} \in\left\{\mathcal{H}(p) \mid p \in \mathbb{P}_{k-1}^{n-1}\right\}$.

(iii),(i) implies (ii): From the proof of the first part we have (i) implies $|\mathcal{H}| \leq\left(\begin{array}{l}n-1 \\ k-1\end{array}\right)$; from that of the second we have (iii) implies $|\mathcal{H}| \geq\left(\begin{array}{l}n-1 \\ k-1\end{array}\right)$.

We are finally in position to call a hypergraph $\mathcal{T}$ that satisfies any two conditions above a linear $k$-tree. The third part of the proof of the theorem hints at two other characterizations of linear $k$-trees.

\section{Theorem 2.4.}

(i) Every $k$-connected hypergraph contains a linear $k$-tree.

(ii) Every linear $k$-forest is contained in a linear $k$-tree.

Proof.

(i): For the sake of contradiction, let $\mathcal{H}$ be a minimal $k$-uniform hypergraph over $X$ that is $k$-connected but does not contain a linear $k$-tree. We let $\mathbb{P}(H)$ represent $\left\{p \in \mathbb{P}_{k-1}^{n-1} \mid\right.$ some $e \in H$ crosses $\left.p\right\}$, where $H \subseteq \mathcal{H}$; we omit braces for singleton arguments. Since $\mathcal{H}$ is not a linear $k$-forest, there is some $e \in \mathcal{H}$ such that $\mathbb{P}(e) \subseteq$ $\mathbb{P}(\mathcal{H} \backslash\{e\})$, hence $\mathcal{H} \backslash\{e\}$ is also a counterexample.

(ii): For the sake of contradiction, let $\mathcal{H}$ be a maximal $k$-uniform hypergraph over $X$ that is a linear $k$-forest but is not contained in a linear $k$-tree. Since $\mathcal{H}$ is not $k$-connected, there is some $p \in \mathbb{P}_{k-1}^{n-1}$ such that $\mathcal{H}(p)=\emptyset$. Let $f \in\left(\begin{array}{l}X \\ k\end{array}\right)$ be some set such that $p\left(\mathbb{1}_{f \backslash\{n\}}\right)=1$, and for $e \in \mathcal{H}$ let $p_{e}$ be a polynomial such that $\mathcal{H}\left(p_{e}\right)=\{e\}$. We set $p_{f}^{\prime}=p$, and for each edge $e \in \mathcal{H}$, we set

$$
p_{e}^{\prime}=\left\{\begin{array}{ll}
p_{e}+p & \text { if } p_{e}\left(\mathbb{1}_{f \backslash\{n\}}\right)=1 \\
p_{e} & \text { otherwise }
\end{array},\right.
$$

which renders $e$ the unique edge in $\mathcal{H} \cup\{f\}$ crossing $p_{e}^{\prime}$ and $\mathcal{H} \cup\{f\}$ a counterexample. 
Thus we may also think of linear $k$-trees as maximal linear $k$-forests or minimally $k$-connected hypergraphs.

\section{$3 \quad$ All trees are not created equal}

A linear $k$-tree is only one of a multitude of possible generalizations of trees to hypergraphs; in this section we explore the connection between linear $k$-trees and a generalization which exists in the literature.

The combinatorial structure known as a $k$-hypertree was introduced in [4] as a tool for developing Bonferroni type inequalities. A $k$-hypertree is a $k$-uniform hypergraph $\mathcal{T}$ on $X$ such that for $k=2, \mathcal{T}$ is a tree with vertex set $X$ and for $k \geq 3, \mathcal{T}$ is defined recursively as follows:

(i) If $X=\{1, \ldots, k\}$ then $\mathcal{T}$ has a unique edge $\{1, \ldots, k\}$.

(ii) If $|X| \geq k+1$ then there exists an element $i \in X$ such that if $e_{1}, \ldots, e_{q}$ denote all edges containing $i$ then $e_{1} \backslash\{i\}, \ldots, e_{q} \backslash\{i\}$ induce an $(k-1)$-hypertree with vertex set $X \backslash\{i\}$ and the remaining edges of $\mathcal{T}$ induce a $k$-hypertree with vertex set $X \backslash\{i\}$. A $k$-hypertree has exactly $\left(\begin{array}{l}n-1 \\ k-1\end{array}\right)$ edges.

The notion was augmented [5] by imposing a total ordering $\mu$ on $X$, yielding several nice characterizations of $k$-hypertrees which generalize properties of trees. We show that linear $k$-trees generalize $k$-hypertrees. We denote the classes of linear $k$-trees and $k$ hypertrees on $X$ by $\mathcal{L} \mathcal{K} \mathcal{T}(k, n)$ and $\mathcal{H} \mathcal{T}(k, n)$ respectively.

Theorem 3.1. $\mathcal{H} \mathcal{T}(k, n) \subset \mathcal{L} \mathcal{K} \mathcal{T}(k, n)$.

Proof. We show inclusion by induction. We have that $\mathcal{H} \mathcal{T}(k, k)=\mathcal{L} \mathcal{K} \mathcal{T}(k, k)$, so let us consider some $\mathcal{T} \in \mathcal{H} \mathcal{T}(k, n)$ for $k<n$. Since $|\mathcal{T}|=\left(\begin{array}{l}n-1 \\ k-1\end{array}\right)$, by Theorem 2.3 it suffices to show $\mathcal{T}$ is $k$-connected. Let $l \in X$ be an element such that $\mathcal{T}_{l}=\{e \backslash\{l\} \mid l \in e \in \mathcal{T}\}$ and $\mathcal{T}_{\bar{l}}=\{e \in \mathcal{T} \mid l \notin e\}$ are respectively $(k-1)$ - and $k$-hypertrees over $X \backslash\{l\}$.

We seek to show that for a polynomial $p\left(x_{1}, \ldots, x_{n-1}\right) \in \mathbb{P}_{k-1}^{n-1}$ there is some edge in $\mathcal{T}=\mathcal{T}_{l} \dot{\cup} \mathcal{T}_{\bar{l}}$ that crosses it. Note that we may assume $l \neq n$ by Lemma 2.2. Dividing by $x_{l}$ gives us $p=x_{l} q\left(x_{1}, \ldots, x_{l-1}, x_{l+1}, \ldots, x_{n-1}\right)+r\left(x_{1}, \ldots, x_{l-1}, x_{l+1}, \ldots, x_{n-1}\right)$. If $r \equiv 0$ then $e \cup\{l\}$ crosses $p=x_{l} q$ for some $e \in \mathcal{T}_{l}$, since $\mathcal{T}_{l} \in \mathcal{K} \mathcal{T}(k-1, n-1)$ by the induction hypothesis. Otherwise some $e \in \mathcal{T}_{\bar{l}}$ crosses $r$, since $\mathcal{T}_{\bar{l}} \in \mathcal{K} \mathcal{T}(k, n-1)$ by the induction hypothesis. In this case $l \notin e$, hence $e$ crosses $p=x_{l} q+r$.

As for strict inclusion, we leave it to the reader to verify that

$$
\begin{array}{r}
\mathcal{T}=\{\{1,2,3\},\{1,2,4\},\{1,2,6\},\{1,4,5\},\{1,5,6\}, \\
\{2,3,5\},\{2,3,6\},\{3,4,5\},\{3,4,6\},\{4,5,6\}\}
\end{array}
$$

is a linear 3-tree but not a 3-hypertree.

The class $\mathcal{L} \mathcal{K} \mathcal{T}(k, n)$ may be a practically significant generalization of $\mathcal{H} \mathcal{T}(k, n)$. Given a cost function $c:\left(\begin{array}{l}X \\ k\end{array}\right) \rightarrow \mathbb{R}_{+}$, it is $N P$-complete to decide whether there is a $k$-hypertree of cost at most $l$ for $n>k \geq 3$ [6]. This is known as the minimum spanning $k$-hypertree 
problem and for $k=2$ reduces to the polynomial time solvable minimum spanning tree problem. Replacing ' $k$-hypertree' with 'linear $k$-tree' in the above definition drastically reduces the complexity of the problem. By Theorems 2.4 and 2.3 the linear $k$-forests on $X$ comprise a matroid, hence we can apply a greedy algorithm to solve the minimum spanning linear $k$-tree problem in polynomial time for constant $k$.

We close by offering a conjecture. A $k$-tree is a $k$-forest of size $\left(\begin{array}{l}n-1 \\ k-1\end{array}\right)$. We let $\mathcal{K} \mathcal{T}(k, n)$ denote the class of $k$-trees on $X$. From Theorem 2.3, Counterexample 2.1, and the fact that $\left\{e \in\left(\begin{array}{l}X \\ k\end{array}\right) \mid 1 \in e\right\} \in \mathcal{H} \mathcal{T}(k, n) \cap \mathcal{K} \mathcal{T}(k, n)$, we derive the following properties.

$$
\begin{array}{lll}
\mathcal{K} \mathcal{T}(k, n) & \subset & \mathcal{L} \mathcal{K} \mathcal{T}(k, n) \\
\mathcal{H} \mathcal{T}(k, n) & \backslash & \mathcal{K} \mathcal{T}(k, n) \neq \emptyset \\
\mathcal{H} \mathcal{T}(k, n) & \cap & \mathcal{K} \mathcal{T}(k, n) \neq \emptyset
\end{array}
$$

Unfortunately these leave the precise interaction of $\mathcal{H} \mathcal{T}(k, n)$ and $\mathcal{K} \mathcal{T}(k, n)$ uncertain. Yet if one could show that for every $\mathcal{T} \in \mathcal{K} \mathcal{T}(k, n)$ there is some $i \in X$ that is contained in exactly $\left(\begin{array}{l}n-2 \\ k-2\end{array}\right)$ edges, then induction would yield the following.

Conjecture 3.2. $\mathcal{K} \mathcal{T}(k, n) \subset \mathcal{H} \mathcal{T}(k, n)$.

\section{Acknowledgements}

The author is grateful to Tom Bohman for exposing him to the tools and the trade.

\section{References}

[1] L. Babai and P. Frankl. Linear Algebra Methods in Combinatorics. Dept. of Computer Science, The Univ. of Chicago, Chicago, 1992.

[2] R. Graham. Personal Communication. 2000.

[3] L. Lovász. Topological and Algebraic Methods in Graph Theory. In Graph theory and related topics (Proc. Conf., Univ. Waterloo, Waterloo, Ont., 1977), pp. 1-14, Academic Press, New York-London, 1979.

[4] I. Tomescu. Hypertrees and Bonferroni inequalities. J. Combin. Theory Ser. B, 41:209-217, 1986.

[5] I. Tomescu. Ordered h-Hypertrees. Discrete Mathematics, 195:241-248, 1992.

[6] I. Tomescu and M. Zimand. Minimum spanning hypertrees. Discrete Appl. Math., 54.1:67-76, 1994. 